\title{
ARTICLE
}

\section{Material discrimination analysis by particle shape of nuclear materials for nuclear forensics application}

\author{
Yoshiki Kimura $^{\mathrm{a}^{*}}$, Nobuo Shinohara ${ }^{\mathrm{a}}$ and Tetsuya Matsumoto ${ }^{\mathrm{b}}$

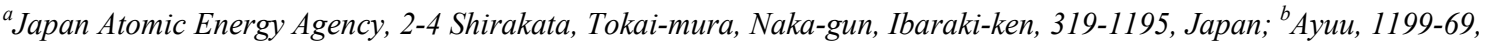 \\ Kobayashi-cho, Mito, Ibaraki, 319-0325, Japan
}

\begin{abstract}
Morphological characteristics of nuclear material is one of important features for material discrimination in nuclear forensic analysis. Japan Atomic Energy Agency (JAEA) has studied the methods and procedures for quantifying particle shapes for nuclear forensics application in order to improve accuracy and speed of nuclear forensic material discrimination. In this paper, current status of the works for material discrimination analysis by particle shapes of nuclear materials for nuclear forensics application performed in JAEA is discussed.
\end{abstract}

Keywords: nuclear forensics; morphology; material discrimination electron microscopy; image analysis; statistical analysis

\section{Introduction}

The Japanese Government issued the national statement at $1^{\text {st }}$ Nuclear Security Summit (Washington D.C., USA) to develop technologies related to measurement of nuclear materials for nuclear forensics, and to share them with the international community, in order to contribute to strengthening the nuclear security system. In response to this statement, Integrated Support Center for Nuclear Non-proliferation and Nuclear Security (ISCN) of Japan Atomic Energy Agency (JAEA) has initiated R\&Ds on nuclear forensics technical capabilities since Japanese Fiscal Year of 2011.

One of the R\&D topics of the nuclear forensics project is to develop the methodology to analyze the morphological characteristics of nuclear materials for the purpose of nuclear forensics application. Morphological characteristics of nuclear material is one of important features for material discrimination (called as nuclear forensics signatures) in nuclear forensic analysis [1,2]. JAEA has been developed the methodologies for capturing particle shapes of nuclear materials from microscope images and material discrimination analysis based on the shape parameters for improvements of accuracy and speed of nuclear forensic material discrimination. In this paper, the current status of the works of JAEA for material discrimination analysis methodology based on particle shape of nuclear materials for nuclear forensics application is discussed.

*Corresponding author. Email: kimura.yoshiki@jaea.go.jp

\section{Capturing of particle shape parameters of nuclear materials}

\subsection{Shape parameters of nuclear material particles}

Nuclear forensics signatures are the material characteristics to identify and discriminate each nuclear materials. It has been well known that the isotope compositions, impurity profiles and material age calculated by parent/daughter ratio would be good nuclear forensics signatures of nuclear materials. On the other hands, morphological signature is comparatively new topic in nuclear forensics, since the quantitative and objective analysis of morphological characteristics by using microscopic image has been technically challenging theme. One reason is that it is very difficult to collect statistically enough numbers of morphological data for material comparison.

JAEA has been focusing on the methodology and procedures to capture shape parameters of nuclear material particles and to utilize them for material discrimination analysis of nuclear forensics. There are two types of parameters to characterize the nuclear materials particles, size and shape parameters (Table 1) [3]. The particle shape parameters could be one of the most useful morphological characteristics of nuclear materials for material discrimination in nuclear forensics. This is because the comparative analysis can be performed by using statistical analysis methodology with enough numbers of morphological data that can be easily captured from limited numbers of microscope images by computational image analysis methodologies. The technical challenges to develop the methodology for nuclear forensics application are; (a) optimized 
procedures to obtain particle images by microscopies including particle sample preparation, (b) capturing (quantification) of particle shape parameters, and (c) material discrimination analytical methodology and strategy based on shape parameters.

Table 1. Shape parameters of nuclear material particles.

\begin{tabular}{ccc}
\hline Size & Category & Terms/Definition \\
& $\begin{array}{c}\text { Area } \\
\text { Perimeter } \\
\text { ECD* } \\
\text { Ellipse Axis }\end{array}$ & $\begin{array}{c}\text { Pixel area, convex full area } \\
\text { Perimeter, convex full perimeter } \\
\text { Diameter of equivalent when circle } \\
\text { Major \& minor axis of } \\
\text { approximate ellipsoid }\end{array}$ \\
\hline Shape & AR & $\begin{array}{c}\text { Ratio of major/minor axis of } \\
\text { approximate ellipsoid, aspect ratio } \\
\text { of bounding rectangle } \\
\end{array}$ \\
& Circularity & $\begin{array}{c}\pi *\left(\text { Pixel Area) } /(\text { Perimeter })^{2}\right. \\
\text { (where circle, Circularity }=1) \\
\text { Catio of pixel area to convex full } \\
\text { Compactness }\end{array}$ \\
& $\begin{array}{c}\text { area } \\
\text { * ECD: Equivalent circular diameter }\end{array}$
\end{tabular}

\subsection{Quantification of particle shape parameters}

JAEA has been developed an original computational software for microscope images to capture the particle shape parameters of nuclear materials, which is named as NF particle image analysis tool. This NF particle image analysis tool has been developed based on an open source computer vision library (Open CV) with the Microsoft Visual $\mathrm{C}++$.

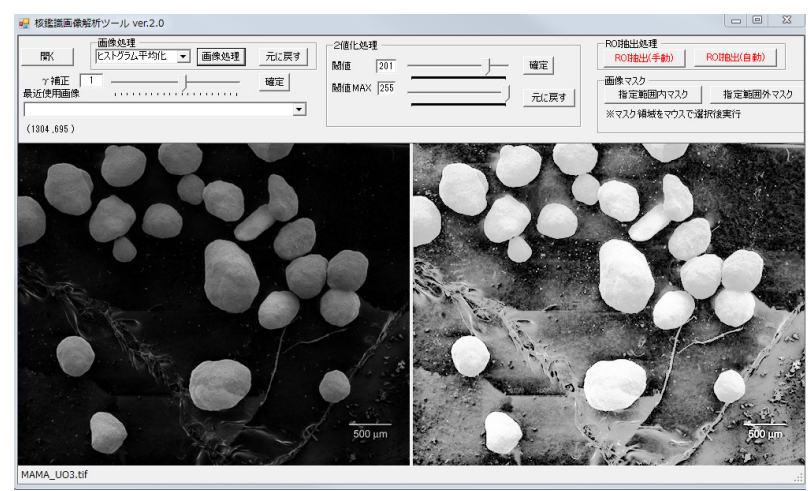

(a) Pre-processing.

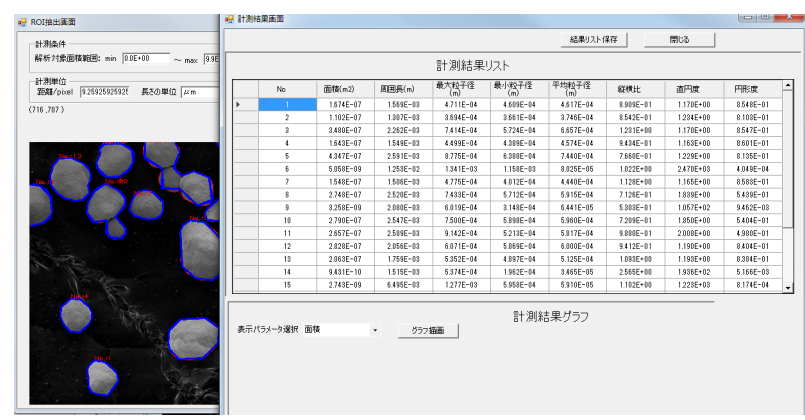

(b) Measurement result.

Figure 1. User interface of NF image analysis tool (SEM image of $\mathrm{UO}_{3}$ ).
Table 2. Process steps and functions of NF image analysis tool developed in JAEA.

\begin{tabular}{ccc}
\hline & Process Step & Functions (Ver.2) \\
\hline 1 & Image capture & $\begin{array}{c}\text { Support Format: } \\
\text {.bmp/.dib/.jpg/.jpeg/.png/.tiff/.tif }\end{array}$ \\
\hline 2 & Pre-processing & $\begin{array}{c}\text { Gamma correction, histogram } \\
\text { flatting, noise removal, edge } \\
\text { detection, masking, monochrome } \\
\text { inversion, watershed (divide } \\
\text { overlaps) }\end{array}$ \\
\hline 3 & Binarization & Monochrome binarization \\
\hline 4 & ROI setting & $\begin{array}{c}\text { Setting of region of interest } \\
\text { (auto/manual) }\end{array}$ \\
\hline 5 & Measurement & $\begin{array}{c}\text { Parameters: shown in Table 1 } \\
\text { Scale setting (manual or scale bar } \\
\text { based), measurement range setting }\end{array}$ \\
\hline 6 & Result display & Table format, histogram view \\
\hline
\end{tabular}

The functions of the tool have been developed to capture the particle shape parameters shown in Table 1, and it supports various formats of gray-scale images obtained from electron microscopes (Table 2). As the first step of image analysis process, users can perform pre-processing on the microscope images to highlight the particles and divide particle overlaps (Figure 1a). The measurements of the particle shape parameters will be performed automatically on the selected region of interest by users after the monochrome binarization process (Figure 1b). The development of the present NF particle image analysis tool has been almost completed, and comparative test with other commercial image analysis software will be performed as the next step.

\section{Material discrimination analysis with particle shape parameters}

\subsection{Analytical methodologies for material discrimination}

The material discrimination analysis is to be performed by comparing distribution of the particle shape parameters between two samples. Three types of analytical methodologies have been tested for the purpose of material discrimination analysis based on shape parameters of nuclear material particles.

(a) K-S test The Kolomogorov-Smirnov two-sample test (K-S test) is a statistical test developed to test whether two distributions are statistically same or different, by quantifying the distance between cumulative distribution functions of the two samples [4]. The $\mathrm{K}-\mathrm{S}$ test is one of the most useful and general nonparametric methods for comparing two samples, since it is sensitive to differences in both location and shape of the cumulative distribution functions of the two samples. The significance level for the K-S test sets to 0.05 in this paper.

(b) Histogram intersection Histogram intersection is an algorithm to calculate the numerical similarity of two histograms easily with possible value of the intersection 
lying between 0 (no overlap) and 1 (completely overlap). This algorithm is used widely as a metric to compare two histograms numerically.

(c) PCA Principal components analysis (PCA) is one of the most standard multivariate analysis methodology. PCA can reduce the dimensions of the multidimensional data and it is primarily used to provide evidence of structure in the data, observe cluster or detect outlier [5].

\subsection{Material discrimination case studies}

Two case studies of material discrimination analysis with nuclear material particle images were performed with the analytical methodologies shown in the previous section. The particle images were analyzed and the shape parameters were captured by the NF particle image analysis tool developed by JAEA.

\subsubsection{SEM image and TEM image of same uranium ore sample}

The particle shape parameters of uranium ore standard material (Cup-2 [7]) captured by two different types of electron microscopes (SEM and TEM) were compared (Figure 2). Distributions of three representative particle shape parameters (ECD, ellipse aspect ratio and compactness) captured by different microscopes were determined as same distributions by KS-test and the numerical similarity was calculated by histogram intersection (Figure 3 and Table 3). It was confirmed that the shape parameters have similar distribution with score plot of PCA (Figure 4, the ellipsoid in the figure shows $99 \%$ probability level of the distributions).

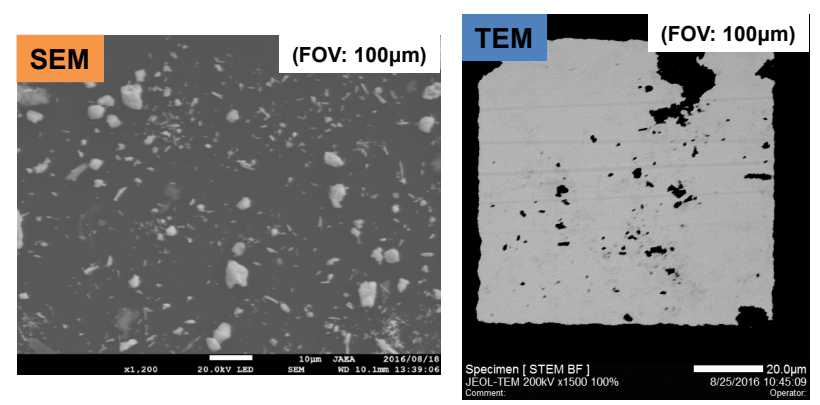

Figure 2. Cup-2 particle images captured by SEM and TEM.

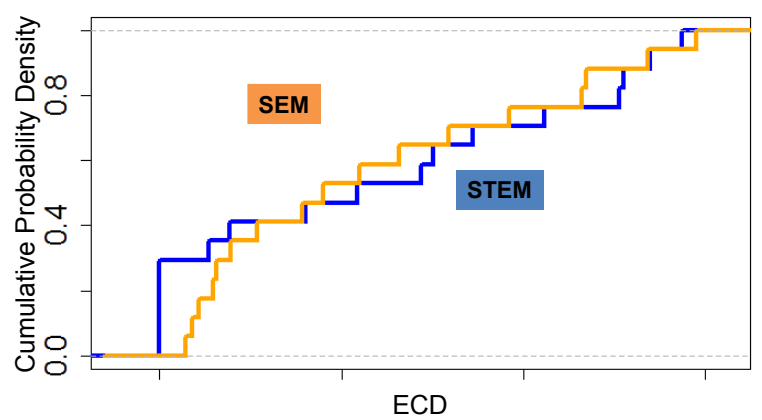

Figure 3. Comparison of cumulative probability density of ECD (Cup-2 image captured by SEM and TEM).
Table 3. Results of KS-test and histogram intersection (Cup-2 image captured by SEM and TEM)

\begin{tabular}{ccc}
\hline Parameter & $\begin{array}{c}\text { KS-test } \\
(\alpha=0.05)\end{array}$ & Histogram Intersection \\
\hline ECD & Match & 0.84 \\
\hline $\begin{array}{c}\text { Ellipse Aspect } \\
\text { Ratio }\end{array}$ & Match & 0.84 \\
\hline Compactness & Match & 0.80 \\
\hline
\end{tabular}

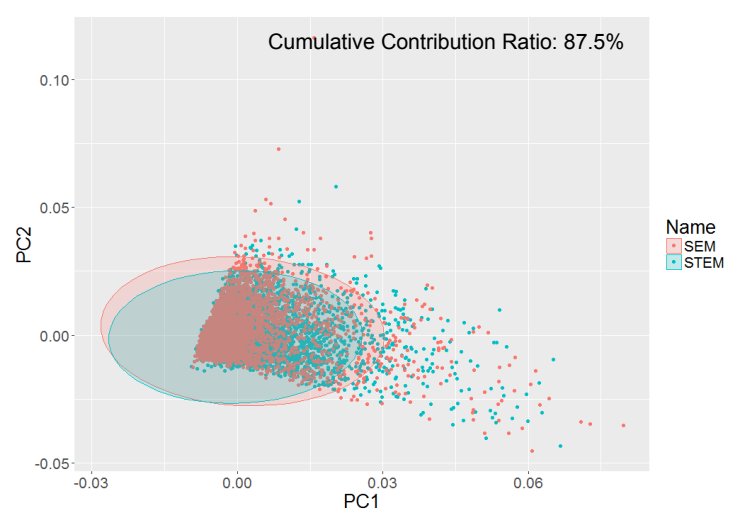

Figure 4. Score plot of PCA analysis (Cup-2 image captured by SEM and TEM).

\subsubsection{SEM image of ammonium diuranate (ADU) and $\mathrm{UO}_{2}$ particles}

The particle shape parameters of two different types of uranium particles, $\mathrm{ADU}$ and $\mathrm{UO}_{2}$, captured by SEM were compared (Figure 5). A difference in ECD, which is a parameter indicating particle size, was detected by KS-test (Figure 6), and its numerical similarity was calculated as 0.59 by histogram intersection (Table 4). The other two parameters were determined as same distributions in this case. It can be determined by these results that the particles of two samples have similar distribution in shapes but different in size. It was also observed that it is difficult to detect difference between the two samples with PCA score plot (Figure 7). PCA can analyze the spread of distribution of multidimensional data with smaller dimensions, but it is difficult to divide samples with large dispersion clearly. However, the morphology parameter to be considered as a significant indicator for material discrimination can be extracted by analyzing factor loadings with PCA methodology.
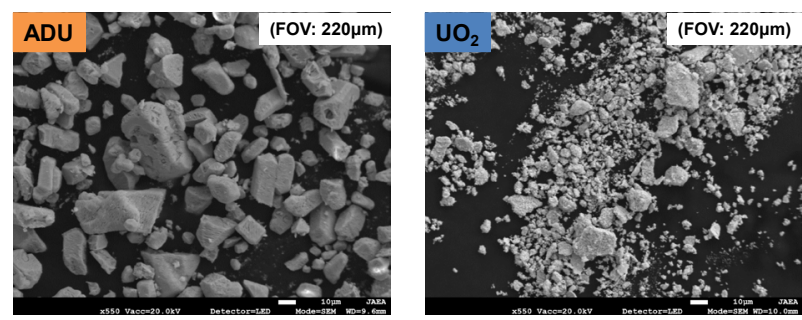

Figure 5. SEM images of $\mathrm{ADU}$ and $\mathrm{UO}_{2}$ particles. 


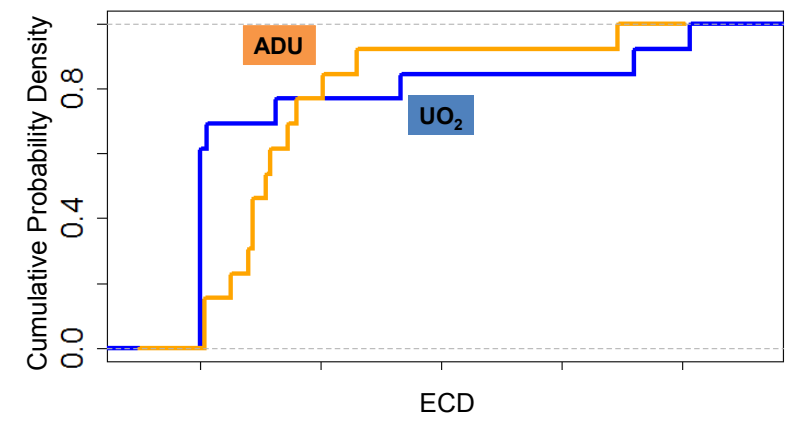

Figure 6. Comparison of cumulative probability density of ECD (SEM images of ADU and $\mathrm{UO}_{2}$ particles).

Table 4. Results of KS-test and histogram intersection (SEM images of $\mathrm{ADU}$ and $\mathrm{UO}_{2}$ particles).

\begin{tabular}{ccc}
\hline Parameter & $\begin{array}{c}\text { KS-test } \\
(\alpha=0.05)\end{array}$ & Histogram Intersection \\
\hline ECD & Not Match & 0.59 \\
\hline $\begin{array}{c}\text { Ellipse Aspect } \\
\text { Ratio }\end{array}$ & Match & 0.90 \\
\hline Compactness & Match & 0.68 \\
\hline
\end{tabular}

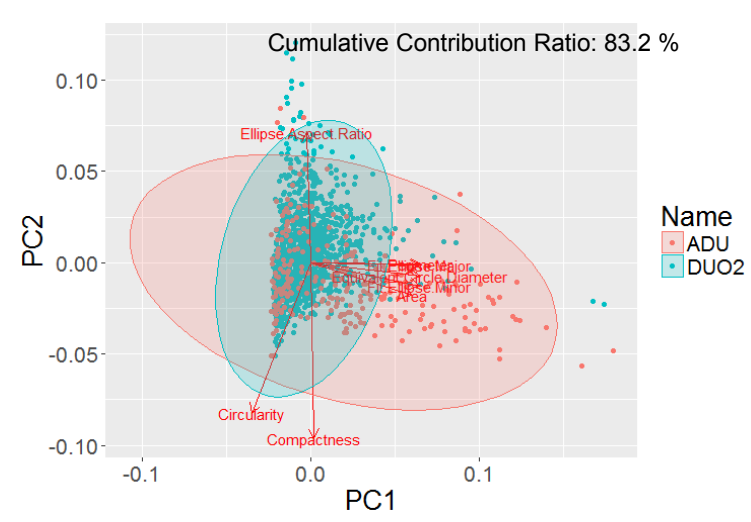

Figure 7. Score plot with factor loadings of PCA analysis (SEM images of $\mathrm{ADU}$ and $\mathrm{UO}_{2}$ particles).

\subsubsection{Summary of two case studies}

It was confirmed that the material discrimination analysis based on particle shape parameters can be performed by comparing the distributions of particle shape parameters with K-S test. Histogram intersection can be also a useful tool to evaluate similarity of the distributions quantitatively. Furthermore, numerical criteria of the similarity to detect different distributions can be estimated as $<0.6$ with histogram intersection (Table 4). This value is just estimated based on the two case studies above so that additional study will be required. It is difficult to detect the differences of two samples using PCA, but it could extract the significant parameter for material discrimination by analyzing loading factors.

\section{Conclusion}

Japan Atomic Energy Agency (JAEA) has studied the methods and procedures of material discrimination analysis based on particle shape for nuclear forensics application for improvements of accuracy and speed of nuclear forensic material discrimination. In this paper, current status of the works for material discrimination analysis by particle shapes of nuclear materials for nuclear forensics application in JAEA was discussed. JAEA has been developed an original computational software for microscope image to capture the particle shape parameters of nuclear materials. The development of the present NF particle image analysis tool has been almost completed, and comparative test with other commercial image analysis software will be performed in next step. Some analytical methodologies were also tested for material discrimination analysis based on particle shape parameters and two case studies with actual nuclear materials were performed.

\section{Acknowledgements}

The work presented in this paper has been supported by the Ministry of Education, Culture, Sports, Science, and Technology (MEXT) of Japan.

\section{References}

[1] IAEA, Nuclear Forensics in Support of Investigations, IAEA NSS 2-G, IAEA (2015).

[2] A.M. Oslen, B. Richards, I. Schwerdt, S. Heffernan, R. Lusk, B. Smith, E. Jurrus, C. Ruggiero and L.W. McDonald, IV, Quantifying morphological features of $\alpha-\mathrm{U}_{3} \mathrm{O}_{8}$ with image analysis for nuclear forensics, Analytical Chemistry 89 (2017), pp. 3177-3183.

[3] C.E. Ruggiero and R.B. Porter, MAMA Software Features: Quantified Attributes, LA-UR-14-23579, LANL (2014).

[4] A. Kolmogoroff, Confidence limits for an unknown distribution function, Annals of Mathematical Statistics 12 (1941), pp. 461-463.

[5] T. Mitsui, S. Okuyama and M. Hida, Qualitative and quantitative analysis using multivariate analysis, BUNSEKI KAGAKU 53 (2004), pp. 773-791. [in Japanese] 